\title{
Providing and Accessing Support During the COVID-19 Pandemic: Experiences of Mental Health Professionals, Community and Vocational Support Providers, and Adults with ASD
}

\author{
Tiffany Thang \\ tthang@ucsc.edu \\ University of California, Santa Cruz \\ Santa Cruz, California, USA \\ Adrian Parrales \\ aparrale@ucsc.edu \\ University of California, Santa Cruz \\ Santa Cruz, California, USA
}

\author{
Alice Liang \\ aliang12@ucsc.edu \\ University of California, Santa Cruz \\ Santa Cruz, California, USA \\ Sara H. Kuang \\ sakuang@ucsc.edu \\ University of California, Santa Cruz \\ Santa Cruz, California, USA \\ Heather Perez \\ hperez@hopeservices.org \\ Hope Services \\ Santa Cruz, California, USA
}

\author{
Yechan Choi \\ ychoi46@ucsc.edu \\ University of California, Santa Cruz \\ Santa Cruz, California, USA \\ Sri Kurniawan \\ skurnia@ucsc.edu \\ University of California, Santa Cruz \\ Santa Cruz, California, USA
}

\begin{abstract}
Due to the COVID-19 pandemic, essential services and support for individuals with ASD have had to transition to telehealth and virtual technologies. While these technologies have been recommended for use to continue provision of essential services to this population, it has yet to be understood what impact it has had on essential service providers and adults with ASD. This experience report provides insight from essential service providers and adults with ASD from a community center for adults with developmental disabilities to understand their experiences in providing and accessing mental health, and community and vocational support during the COVID19 pandemic through telehealth and virtual technologies.
\end{abstract}

\section{CCS CONCEPTS}

- Human-centered computing $\rightarrow$ Accessibility.

\section{KEYWORDS}

autism spectrum disorders, ASD, COVID-19, essential services, mental health, community centers, telehealth, virtual conferencing

ACM Reference Format:

Tiffany Thang, Alice Liang, Yechan Choi, Adrian Parrales, Sara H. Kuang, Sri Kurniawan, and Heather Perez. 2021. Providing and Accessing Support During the COVID-19 Pandemic: Experiences of Mental Health Professionals, Community and Vocational Support Providers, and Adults with ASD. In The 23rd International ACM SIGACCESS Conference on Computers and

This work is licensed under a Creative Commons Attribution International 4.0 License.

ASSETS '21, October 18-22, 2021, Virtual Event, USA

(c) 2021 Copyright held by the owner/author(s).

ACM ISBN 978-1-4503-8306-6/21/10.

https://doi.org/10.1145/3441852.3476470
Accessibility (ASSETS '21), October 18-22, 2021, Virtual Event,USA. ACM, New York, NY, USA, 6 pages. https://doi.org/10.1145/3441852.3476470

\section{INTRODUCTION}

Autism spectrum disorders (ASD) are a developmental disability affecting 1 in 47 adults in the United States [2]. Many of these individuals will also experience co-occurring mental health conditions, such as anxiety, depression, and Post Traumatic Stress Disorder (PTSD), often as a result of traumatic experiences involving bullying or discrimination due to their condition $[4,7,10]$. Research has also suggested that adults with ASD will often experience greater rates of unemployment or under-employment, along with limited opportunities for community or social activities [2]. Community centers for adults with ASD have risen as a method of helping this population with addressing their mental health, vocational and social needs, through providing access to mental health professionals, job coaches, and opportunities for them to be involved within their community.

Due to the COVID-19 pandemic, in-person support provided by community centers ceased in some states to protect the health of staff and clients, although research suggests that support for ASD populations was much needed. Current research evaluating the impacts of the COVID-19 pandemic on populations of individuals with ASD emphasize increased feelings of anxiety and negative mental health symptoms as a result of the rapid and unpredictable nature of the pandemic $[3,6,8]$. In a survey of parents of children with ASD, it was found that the pandemic had resulted in increased difficulty for families in managing daily routines and instances of difficult behaviors presented by their children [3]. A similar study involving a survey of adolescents with ASD and their caregivers found increased instances of irritability, social withdrawal and poor sleep quality for adolescents and increased anxiety for caregivers during this time [8]. Additional studies have emphasized the need 
to support individuals with ASD, as the pandemic imposes feelings of uncertainty and distress, which has been shown to intensify ASD symptoms and worsen co-occurring mental health conditions [9].

As the need for support during the pandemic is evident through this research, studies have suggested that telehealth and virtual technologies be used as a tool to provide continued and accessible support to individuals with ASD $[1,11]$. Evaluations of this technology have been conducted with caregivers of children with ASD $[6,13]$, but research has yet to examine the impact of telehealth on support for adults with ASD. In particular, the impact of telehealth and the experiences of essential service providers for adults with ASD, and adults with ASD during the pandemic has yet to be explored.

In this experience report, we detail findings from interviews with essential service providers from Hope Services, a community center for adults with developmental disabilities with branches across California, to gain an understanding of their experiences providing services to clients during the COVID-19 pandemic. Interview with clients of Hope Services will also be described to highlight their experiences with accessing services and receiving support during this time. Through this report, we hope to provide insight as to how the pandemic has impacted these essential service providers and adults with ASD, and aim to suggest improvements to telehealth and related technologies to better accommodate for their needs.

\section{EXPERIENCES}

This report details the experiences of 10 essential service providers from Hope Services who worked with clients during the COVID19 pandemic. Mental health professionals, job coaches and programs coordinators were among the essential service providers interviewed, with some supporting clients through telehealth or virtual conferencing technologies, such as Zoom (https://zoom.us), and some continuing providing services in-person when necessary. Those who continued providing services in-person followed social-distancing protocols and safety measures. Information regarding their experiences providing services during the pandemic were collected through interviews with questions focused on understanding the ways in which the provision of services had changed, what technologies were being used to connect them with clients and how their experiences have shaped their feelings towards providing services remotely. Essential service providers were recruited from two branches of Hope Services that serviced suburban and rural areas south of the Bay Area of California. They described working with clients in their early 20 s to clients in their 60 s and 70 s. Providers stated that their clients were predominantly male, with one reporting that the average gender demographic of their branch was $70 \%$ male and $30 \%$ female. Essential service providers also described working with clients of diverse ethnicities, including those of Asian, African, and Latin American descent, although some did note that the majority of their clients were Caucasian.

In addition to interviews with essential service providers, interviews with 4 clients of the community center were conducted to gain an understanding of their experiences with accessing services remotely and their feelings towards the pandemic. Interviews with essential service providers and clients were conducted approximately a year after the start of the pandemic and continued through the beginning of vaccine distribution to essential workers and at-risk populations in the United States. Interviews were coded by 4 undergraduate students using thematic analysis, to identify common themes or patterns that arose within each set of interviews. The following sections will describe findings amongst interviews with essential service providers and clients of Hope Services.

\subsection{Essential Service Provider Interviews}

Essential service providers interviewed included Job Coaches, Programs Coordinators, Community Support Facilitators, and mental health professionals, such as Mental Health Clinicians, Licensed Marriage and Family Therapists (LMFT), and Clinical Social Workers. Mental health professionals worked with clients from the community center on managing co-occurring mental health conditions, including depression and anxiety. Job Coaches, Programs Coordinators and Community Support Facilitators provided support to clients by providing them vocational resources and opportunities for employment, connecting clients with volunteer opportunities throughout their communities, and creating social and educational opportunities. These essential service providers will collectively be referred to within this report as community and vocational support providers. Of the 10 essential service providers interviewed, 5 were mental health professionals and 5 were community and vocational support providers.

2.1.1 Session Time. Prior to the COVID-19 pandemic, these essential service providers all worked with clients in-person, either at their homes, the community center, or sites within the community. Mental health professionals described working with 21 clients a month on average, where sessions with clients would typically be held once a week. These sessions would vary in length, being anywhere from 30 minutes to an hour depending on the needs of the client. Community and vocational support providers would work with an average of 35 clients a month and provided enrichment classes, community volunteer activities, and work opportunities, anywhere from once a week to 5 days a week. Each day would vary in the amount of time spent with a client, as classes could be between 2 and 3 hours, whereas volunteer and work opportunities would often encompass a full 8-hour day.

During the pandemic, these essential service providers noted changes with respect to the amount of time spent working with clients. While some transitioned to providing services remotely, others continued to provide in-person services that could not be translated to a remote format. Mental health professionals who continued to provide support to clients in-person noted an increased amount of time spent with clients, going from 30 minute sessions pre-pandemic to 1 hour and 15 minute sessions during the pandemic. Professionals attributed these increases to social distancing and shelter-in-place ordinances that deprived clients of opportunities for in-person interactions. They also wanted to ensure that they effectively supported clients in dealing with the ongoing stressors of the pandemic, leading to increased session times. Mental health professionals working remotely noticed an increase in the number of sessions held per client after the onset of the pandemic. Typically 
they met with clients once a week, but after the pandemic began these professionals began to meet with clients 2-3 times a week to ensure that they felt supported and received help in coping with the uncertainty and stress of the pandemic. While the number of sessions per week increased, it was noted that the amount of time per session would increase or decrease based on each client's level of comfort with telehealth and availability of stable internet connection. A mental health professional noted that time with one of their clients had increased as a result of the client feeling too comfortable with the telehealth medium. They stated this client would call them 15-20 times for non-emergency purposes after the transition to telehealth sessions over the phone, suggesting that "the phone call medium has also been challenging because it adds a layer of informality", leading to some difficulty setting boundaries and managing session times.

During the pandemic, community and vocational support providers worked with a similar number of clients on average, but the capacity in which they worked with them had changed. Some of the job sites that clients worked in were closed due to the pandemic, resulting in a shift to focusing on classes and coursework. Community and vocational support providers led distance learning classes over Zoom to ensure that clients continued to have the opportunity to learn new skills and interact with their peers. These classes were shorter than those that were held in-person, but this change was to encourage clients to spend some time away from their screens to promote there physical and mental well-being. Providers working with job sites that remained open during the pandemic found that the number of clients they worked with was the same, but some clients would come to work less frequently. Even though necessary precautions and protocols were in place to protect them, providers noted that clients feared the possibility of becoming sick with COVID-19 while working.

Essential service providers note changes with respect to time spent with clients as a result of the pandemic and the switch to telehealth. Mental health professionals continuing to work in-person describe increases in session times, while those providing services through telehealth saw an increase in the number of sessions per week, but variability in session times. Community and vocational support providers primarily transitioned to providing services remotely, with a decrease in time spent with clients. For those continuing to work in-person at job sites with clients, they note that clients have chosen to decrease the amount of days they spent working on-site due to fears of contracting COVID-19. While changes in session times and time spent with clients are not identical across essential service providers' experiences, these findings stress that the pandemic and related changes can impact everyone differently, requiring flexibility across accommodations and services provided to individuals with ASD. These interviews provide some insight as to how essential service providers have altered their session times to better accommodate for the needs of their clients, and identify client's level of comfort with telehealth, internet connectivity, and COVID-19 related stressors as factors contributing to changes in session times.

2.1.2 Technical Issues. During the pandemic, Hope Services distributed tablets to its clients to ensure services could continue to be provided remotely over Zoom. As a result, essential service providers of the community center have seen an increase in the use of technology in their work. While they describe an appreciation for the technology for allowing their work with clients to continue remotely, they emphasize that it is not without fault. Towards the beginning of the pandemic, essential service providers noted issues with installing and running Zoom and mentioned that this would often impact their ability to take advantage of allotted session times with clients. Providers also described their clients having these same issues, where one mentioned that it took clients "a long time to learn how to use Zoom, [for] some people up to six months". In addition to this, some providers described the initial transition to telehealth and remote services as challenging, stating that more training on how to use the technology would have been helpful. Providers also described a desire for greater understanding of how to take advantage of Zoom's features for their work, wanting access to more tools and games to use with clients. As one provider stated, "if I had more access to [trainings] that'd be helpful, but I'm hoping to kind of transition out of telehealth", representing the feelings of many of the providers interviewed, who would prefer to continue providing services in-person.

A community and vocational support provider also described anticipating technical issues during their meetings with clients, where glitches and internet connectivity issues were expected to occur at some point during any of their meetings. These were a common issue amongst the essential service providers interviewed, as many stated that unstable internet on both their end and on the client's end often interfered with their ability to provide services. A mental health professional described needing to provide services over the phone as opposed to Zoom due to their poor internet connectivity at home, mentioning that their "internet connection is not reliable ... so there was a lot of driving to a place where [they] had regular cell service and having to sit and do session in [the] car". During wildfire season in California, providers described their experiences being evacuated from their homes and losing power, while still trying to stay connected with their clients. Providers struggled to provide telehealth sessions in hotel rooms with unstable Wi-Fi. In one account, a mental health professional described losing power in their home and conducting sessions over the phone. They would "drive around for awhile ... with a plug [next to] the cigarette lighter" to charge their phone and ensure they would be able to continue providing support to their clients.

Essential service providers were determined to continue providing support to their clients during the COVID-19 pandemic, even though they faced many technical issues with Zoom and reliable internet connection. Providers described lack of telehealth training as one of the main issues they faced at first, without much knowledge of how to take advantage of the technology for their work with clients. Unreliable internet connection also heavily impacted providers' ability to conduct services remotely, requiring some to drive around and hold sessions over the phone in their cars. California's wildfire season intensified the situation, where power outages and evacuations made access to stable internet much more difficult. While these essential service providers have demonstrated ways in which they have accommodated for these challenges, the need for accessible and reliable home internet services is emphasized. 
Essential service providers suggest that increased telehealth training may have alleviated issues they faced in providing services remotely, describing difficulty in using Zoom to work with clients. Although more training may help with using these technologies, these findings suggest that the development of telehealth technologies specifically catered to these professionals would be beneficial. Current telehealth technologies lack the tools and features they need to provide services to their clients, leaving many eager to transition back to providing services in-person.

2.1.3 Stress. As described in the previous section, many providers found the transition to telehealth and remote services to be challenging, especially with respect to adapting their regular activities and materials to a virtual format. Difficulty understanding how to use these technologies to provide services to clients and lack of tools and features resulted in increased levels of stress for essential service providers. A mental health professional described experiencing a great amount of stress and fatigue, stating that they "don't know if [they] have the tools [they] need to provide effective services to clients". Many essential service providers discussed wanting to maintain the same quality of services they would normally provide in-person over Zoom, but became frustrated when this proved to be a difficult task.

As suggested in the previous section, increased telehealth trainings could have been a potential solution to this issue, but these findings also suggest that telehealth in its current state does not have the ability to effectively help translate some of these services to a virtual format. While essential service providers describe attempts to translate their work to virtual formats in ways that maintain its quality for clients, telehealth's lack of specialized features and tools for this population of users may be preventing this from happening. This suggests that the development of more specialized telehealth technologies may relieve some of the stress and better serve the interests of its users.

2.1.4 Connectedness. Despite not being able to meet with clients and co-workers in-person, essential service providers were able to maintain a sense of connectedness as they provided remote services. Through using Zoom, providers were able to connect with other providers, and have their clients connect with other clients from different branches of the organization. In doing this, they were able to meet and socialize with new people, that they otherwise would rarely interact with, on a regular basis. A community support provider described the experience as "a fun relaxing time, and everyone's talking ... they just light up and talk, even the clients that aren't used to saying that much".

At the same time, there was a sense of disconnection, where providers stated that not being able to see clients who have their camera off in Zoom led them to wonder whether the client was truly engaging with them, and it made it more difficult to understand how the client was feeling. Among mental health professionals who met clients in-person, there were instances where disconnection was also prevalent. A mental health professional described not being able to see, "small expressions that [clients] are making [while they're wearing their mask] and ... their non-verbal communication", making it harder to get an accurate sense of their well-being.

While essential service providers described being able to use Zoom to maintain a feeling of connectedness with their clients and co-workers, there were feelings of disconnection experienced between essential service providers and their clients during sessions together. Disconnection was attributed to the loss of non-verbal, visual cues which enabled providers to get a more accurate sense of their clients' engagement and well-being. This finding acknowledges that telehealth and virtual platforms can keep individuals connected in a broader sense, but for these essential service providers to effectively support their clients with ASD, more effort is needed to accurately gauge their well-being and engagement.

\section{CLIENT INTERVIEWS}

There were 4 clients of Hope Services that were interviewed regarding their experiences with the pandemic. Interview questions focused on understanding their experiences with accessing support services, socializing with others, and using technology during the pandemic. Clients interviewed lived in suburban or rural areas and received services remotely throughout the duration of the pandemic. This section will describe findings from interviews with clients, followed by a discussion on the implications of findings from interviews with essential service providers and clients.

3.0.1 Social Interactions. Clients expressed a desire to return to in-person interactions, stating their preference for in-person interactions over interactions in Zoom. One client stated that they "really want to go back [to Hope Services] and see all the people", with another mentioning that they are "looking forward to meeting up with [their] friends and family in-person". While clients demonstrated a preference for in-person social interactions, they were able to increase their overall social interactions remotely, using technology such as Zoom and social media, to socialize with their peers and providers at the community center, and their family and friends. Prior to the pandemic, clients described seeing their friends in-person about once a week and family on special occasions. However, during the pandemic, clients found themselves connecting with loved ones through social media, and video and phone calls more frequently. In addition to this, clients appreciated that they were able to stay connected to staff and peers at Hope Services through accessing services through Zoom and felt supported by the essential service providers they worked with.

3.0.2 Feelings Towards Technology. During the pandemic, clients described their experience with transitioning to remote services by accessing meetings with staff at the community center through Zoom. Clients described the initial transition as difficult, due to the lack of technical resources available to them. Hope Services remedied this issue by distributing tablets to clients, but issues regarding internet connection arose. Some clients described their experience with accessing Zoom as follows, "I kind of had a difficult time trying to install Zoom on my tablet... we have a crazy internet connection, I would have difficulty sometimes getting logged in”.

Even with these initial issues, clients did mention feeling appreciative towards technology in allowing them to stay connected to others. Clients preferred in-person services, but many were pleased by the ability to keep in touch with others through technologies such as Zoom and FaceTime. A client affected by the wildfires described using Zoom to keep in touch with friends and staff at the community center, "my family had to evacuate with the wildfires 
from our home ... staying on Zoom has helped me stay in contact with friends and staff from Hope". Another client described moving to a new city during the pandemic, and stated that "it's nice to see some good friendly faces on Zoom that I can see every day ... even though it's remotely, I'm glad to see them".

3.0.3 Hope for the Future. After experiencing the pandemic for about a year, clients remained hopeful that they would once again be able to interact with their friends and providers at Hope Services. Clients described how COVID-19 has impacted their lives, discussing how they noticed changes in their households in addition to the changes with Hope Services. A client described "crazy restrictions" implemented anytime they went to the grocery store with their mother to buy essentials. Another client stated that it was "really hard to wear a mask every day" and described being hesitant to interact with friends in-person because of this. Clients all described a strong desire for the pandemic to end, especially since they were unable to access some of the services that could not be transitioned to a remote medium. However, clients are optimistic, with one mentioning that they "registered [their] mom and [themselves] for the vaccine" and were looking forward to "seeing [their] friends again". Another client stated that they were "actually glad [scientists] came up with [the] vaccine because there's a real hope ... [of] see[ing] each other in-person again".

\section{DISCUSSION AND CONCLUSION}

Interviews with essential service providers and clients of Hope Services have helped provide some insight as to how support for adults with ASDs has continued during the pandemic. While these interviews only provide a glimpse into the experiences of adults with ASD and their essential service providers, they allow for an understanding of how telehealth and related technologies are being used to continue support services during the pandemic, and how they may improve to better support these populations in the future.

Our findings detail telehealth as a challenging medium, where difficulty transitioning to providing services in a virtual format due to lack of necessary tools and features of the technology were emphasized. While providers attempted to adapt to these technologies, the lack of training, features and tools, and unstable internet connectivity led to increased levels of stress and frustration. Clients describe the same difficulty in transitioning to telehealth, with lack of technological resources and reliable internet service as barriers to accessing support. Even so, these interviews suggest that they were able to stay connected with friends and family through virtual platforms and were optimistic about the future, with many eager to see friends, family and essential service providers in-person once again.

Findings from these interviews suggest that telehealth technologies may further benefit from providing more specialized features and tools for specific user groups. This is consistent with additional research regarding the use of telehealth for individuals with ASD, where studies have suggested the lack of features for providers results in less effective care and support [1], and leads to a preference for in-person services [13]. These findings also suggest that access to reliable internet service is important in ensuring that individuals with ASD may continue to receive support through telehealth during the pandemic, where both essential service providers and clients were impacted by lack of stable internet access. Consistent with other findings $[5,12]$, this suggests that increasing access to reliable at-home internet services is crucial to supporting the provision of effective support through telehealth.

Although the pandemic draws closer to an end, telehealth may continue to provide support to individuals with ASD, especially in regions where in-person support is not accessible. This report provides insight as to how telehealth and virtual technologies have been used by essential service providers and adults with ASD in continuing access to mental health, and vocational and community support services during the COVID-19 pandemic. Based on their experiences, we suggest future work focus on developing telehealth and virtual technologies with stakeholders to ensure the tools and features needed by users are included, and to allow a smoother transition from in-person to telehealth or virtual services. Ultimately, future research should continue to examine the impact of telehealth and virtual technologies on ASD populations and essential service providers during the COVID-19 pandemic to determine how these technologies may improve.

\section{REFERENCES}

[1] Stephanie H. Ameis, Meng-Chuan Lai, Benoit H. Mulsant, and Peter Szatmari. 2020. Coping, fostering resilience, and driving care innovation for autistic people and their families during the COVID-19 pandemic and beyond. Molecular Autism 11, 1 (2020). https://doi.org/10.1186/s13229-020-00365-y

[2] CDC. 2020. Autism Spectrum Disorder in Teenagers \& adults. https://www.cdc. gov/ncbddd/autism/autism-spectrum-disorder-in-teenagers-adults.html

[3] Marco Colizzi, Elena Sironi, Federico Antonini, Marco Luigi Ciceri, Chiara Bovo, and Leonardo Zoccante. 2020. Psychosocial and Behavioral Impact of COVID-19 in Autism Spectrum Disorder: An Online Parent Survey. Brain Sciences 10, 6 (2020). https://doi.org/10.3390/brainsci10060341

[4] Sally-Ann Cooper, Elita Smiley, Jillian Morrison, Andrew Williamson, and Linda Allan. 2007. Mental ill-health in adults with intellectual disabilities: prevalence and associated factors. The British fournal of Psychiatry: The fournal of Mental Science 190 (2007), 27-35. https://doi.org/10.1192/bjp.bp.106.022483

[5] Lauren Franz, Jill Howard, Marisa Viljoen, Linmarie Sikich, Tara Chandrasekhar, Scott H. Kollins, Lawrence Lee, Minkateko Ndlovu, Maura Sabatos-DeVitto, Noleen Seris, Nokuthula Shabalala, Marina Spanos, Petrus J. de Vries, and Geraldine Dawson. 2021. Pragmatic adaptations of telehealth-delivered caregiver coaching for children with autism in the context of COVID-19: Perspectives from the United States and South Africa. Autism (2021). https: //doi.org/10.1177/13623613211022585

[6] Carla B. Kalvin, Rebecca P. Jordan, Sonia N. Rowley, Anna Weis, Karen S. Wood, Jeffery J. Wood, Karim Ibrahim, and Denis G. Sukhodolsky. 2021. Conducting CBT for Anxiety in Children with Autism Spectrum Disorder During COVID19 Pandemic. Fournal of Autism and Developmental Disorders (2021). https: //doi.org/10.1007/s10803-020-04845-1

[7] Mario G. Mazza, Aurora Rossetti, Giovanna Crespi, and Massimo Clerici. 2020. Prevalence of co-occurring psychiatric disorders in adults and adolescents with intellectual disability: A systematic review and meta-analysis. Fournal of Applied Research in Intellectual Disabilities 33, 2 (2020), 126-138. https://doi.org/10.1111/ jar.12654

[8] Tuba Mutluer, Ceymi Doenyas, and Herdem Aslan Genc. 2020. Behavioral Implications of the Covid-19 Process for Autism Spectrum Disorder, and Individuals' Comprehension of and Reactions to the Pandemic Conditions. Frontiers in Psychiatry 11 (2020). https://doi.org/10.3389/fpsyt.2020.561882

[9] J. A. Patel, A. A. Badiani, F. B. H. Nielsen, S. Assi, V. Unadkat, B. Patel, C. Courtney, and L. Hallas. [n.d.]. COVID-19 and autism: Uncertainty, distress and feeling forgotten. Public Health in Practice 1 ([n.d.]). https://doi.org/10.1016/j.puhip. 2020.100034

[10] Freya Rumball, Kinga Antal, Francesca Happé, and Nick Grey. 2021. Co-occurring mental health symptoms and cognitive processes in trauma-exposed ASD adults. Research in Developmental Disabilities 110 (2021). https://doi.org/10.1016/j.ridd. 2020.103836

[11] Dasha Solomon and Neelkamal Soares. 2021. Telehealth Approaches to Care Coordination in Autism Spectrum Disorder. In Interprofessional Care Coordination for Pediatric Autism Spectrum Disorder: Translating Research into Practice, Maryellen Brunson McClain, Jeffery D. Shahidullah, and Katherine R. Mezher (Eds.). Springer International Publishing, 289-306. https://doi.org/10.1007/9783-030-46295-6_19 
[12] Liliana Wagner, Laura L. Corona, Amy S. Weitlauf, Kathryn L. Marsh, Anna F. Berman, Neill A. Broderick, Sara Francis, Jeffrey Hine, Amy Nicholson, Caitlin Stone, and Zachary Warren. 2020. Use of the TELE-ASD-PEDS for Autism Evaluations in Response to COVID-19: Preliminary Outcomes and Clinician Acceptability. Fournal of Autism and Developmental Disorders (2020). https: //doi.org/10.1007/s10803-020-04767-y

[13] Susan W. White, Laura Stoppelbein, Hunter Scott, and Debbie Spain. 2021. It took a pandemic: Perspectives on impact, stress, and telehealth from caregivers of people with autism. Research in Developmental Disabilities 113 (2021). https: //doi.org/10.1016/j.ridd.2021.103938 